\title{
Comments on: Multicriteria decision systems for financial problems
}

\author{
David Pla-Santamaria • Ana Garcia-Bernabeu
}

Published online: 4 May 2013

(C) Sociedad de Estadística e Investigación Operativa 2013

Well written and organized, the paper by Zopounidis and Doumpos suggests to us the following questions: (a) whether or not the multiple criteria decision aid (MCDA) approaches are realistic and convincing in environments of Simon's bounded rationality; (b) whether or not the MCDA approaches rely on assumptions which are in accordance to (or at least, are not in contradiction to) principles firmly accepted in traditional financial theory and economics. If questions (a)-(b) have a positive answer, then we can expect that sooner or later, MCDA approaches will be incorporated into the traditional finance textbooks. If questions (a)-(b) have a negative answer, then disagreement and mutual overlooking would be inevitable.

Here after, quotation marks without author's name are sentences from the paper by Zopounidis and Doumpos.

\section{First question: realism and applicability}

Models in traditional financial theory are often realistic but sometimes are not. For example, capital asset pricing model (CAPM) "is developed in a hypothetical world" with hypotheses that might be not sufficiently realistic, such as there exists a risk-free asset such that investors may borrow or lend unlimited amounts at the risk-free rate (Copeland and Weston 1988, p. 194).

Not all but most MCDA approaches to finance are realistic. "The finance theory has adopted the wealth maximization principle, focusing on normative and descriptive approaches often highlighting multiple factors that drive this single goal.

This comment refers to the invited paper available at doi:10.1007/s11750-013-0279-7.

D. Pla-Santamaria $(\bowtie) \cdot$ A. Garcia-Bernabeu

Universitat Politècnica de València, Plaza Ferrandiz y Carbonell, 03801 Alcoy, Spain

e-mail: dplasan@upv.es 
Multicriteria decision systems add important practical contributions in this context, supporting financial decision makers in modeling, analyzing, and evaluating multiple ways of action, under all decision criteria pertinent in a specific decision instance [...]. Compared to the traditional operations research paradigm, which is based on a single objective maximization framework, MCDA models are built considering all aspects of a given financial decision problem" (p. 1 and pp. 2-3). Certainly, wise investors, or generally speaking, wise people make their decisions after considering the multiple aspect of the problem, circumstances and implications. Several goals are pursued by the investor to reach different targets. As a solution satisfying all the investor's aspirations is an infeasible solution, the investor looks for a compromise among goals. Multiple favorable or adverse scenarios and events can happen and they are evaluated by the investor from believes, guesses, and in terms of likelihood. Due to this complexity of human decisions, MCDA better fits human nature (often full of inconsistency) than the wealth maximization principle does.

Zopounidis and Doumpos point out the difference between normative (or prescriptive) models and descriptive models. We agree with them about it. "Bouyssou et al. define the prescriptive approach as one that discovers models suitable for a given decision maker in a particular decision context, on the basis of information gathered on his/her system of values" (p. 9). Both MCDA and classical financial models can be viewed as nice normative rules whatever their descriptive value if their practical consequences are positively tested.

There is an ongoing issue with applicability. So far, banks and monetary institutions are using MCDA in a very limited way. This fact cannot be merely explained by difficulties of managing mathematical models. Indeed, this is a fact related to habits and education. Business schools, finance schools, and faculties of economics have educational programs, which are intensive in accounting and standard financial methods, but rather poor in operational research techniques. Banks and monetary institutions live in an accounting world. Their strategies are built from empirical information, brilliant ideas, marketing rules and political background. Fund managers and financial consultants escape this world, and frequently use MCDA techniques. Probably, MCDA can help banks and managers improve their outcomes in a significant way but a long effort is needed to persuade them about it. As to bank and credit rating, see Doumpos and Zopounidis (2010, 2011).

\section{Second question: assumptions and appropriate use of models}

Approaching financial problems by MCDA models in a mechanical way (namely, without analyzing soundness and appropriateness from financial assumptions) is not advisable. "MCDA approaches are based on ex-ante verification of the models' structural hypotheses combined with sensitivity and robustness analysis. Applying sound validation procedures to MCDA models on the basis of principles widely accepted by finance practitioners and regulators, increases their success potential and their adoption in practice (p. 16)." Sometimes, nonfinancial models (namely, models aimed at solving specific problems in technical areas such as industrial engineering) are brought to the financial field and are there mechanically used. This transfer is valid if 
and only if reliable financial assumptions are stated to support the new use. For example, Data Envelopment Analysis (DEA) allows to determine efficient frontiers from technological assumptions. To use this method for the purpose of ranking financial investments would require reformulating DEA on a financial axiomatic basis. Notice the following difficulties: (a) how to strictly characterize the financial criteria (e.g., volatility, beta parameter, liquidity, diversification, etc.) as DEA inputs and outputs; (b) how to deal with the investor's preferences; (c) how to deal with Arrow's risk aversion which depends on the investor's preferences; (d) how to rank the efficient alternatives taking into account that the various MCDA approaches proposed in the literature are not financial-based approaches and lead to rankings which substantially differ from one another. See comparison of results in Sun et al. (2005).

\section{The case of portfolio selection}

A first point to be commented concerns outranking methods to screen opportunity sets. "In a multicriteria context, the modeling of the stock selection process was first introduced by Hurson and Zopounidis who proposed the use of outranking and disaggregation techniques, combined with a MOO model. In particular, an outranking technique (ELECTRE TRI method) and the UTASTAR disaggregation model were first used to select a limited set of stocks on the basis of financial and stock market criteria" (p. 11). In practice of portfolio selection, most opportunity sets are large. Then pairwise comparison in outranking methods such as analytic hierarchy process (AHP), lead to cumbersome (almost infeasible) processes. Moreover, methods such as AHP can provide unreliable results if the number of pairwise compared variable is high. See Saaty and Ozdemir (2003). Therefore, AHP should not be used in scenarios with many variables. A second point concerns "mean absolute deviation model (Konno and Yamazaki 1991)." We wonder if financial theory based on the mean-variance $(\mathrm{M}-\mathrm{V})$ paradigm will accept mean absolute deviation as a proxy for portfolio variance. The Konno and Yamazaki's argument to justify their proposal is that $\mathrm{M}-\mathrm{V}$ model is quadratic and, therefore, is quite difficult to solve, especially for large problems. We do not agree with this opinion. Notice that the paper by Konno and Yamazaki is very old (1991). Currently, available software has improved in a substantial way, so that Matlab or Lingo (GENPRT.lg4) software easily solves M-V large scale optimization problems.

Finally, the role of Arrow's (1965, p. 94), risk aversion in the portfolio choice problem should be examined. "In a normative context, specific utility functions (of wealth) are assumed to model risk aversion. For instance, Markowitz's mean-variance model implicitly assumes a quadratic utility function (for examples and an analysis of other utility forms see). However, a DM's attitude toward risk is inevitably subjective and it is connected to the utility of the alternatives under consideration. As a consequence, general risk models grounded on financial and economic principles should be combined with operational techniques providing individualized decision support in the context of a specific financial problem and the risk attitude of a particular DM" (p. 8). Indeed, investor's risk aversion should influence the portfolio selection process. Two investors facing the same opportunity set with equal return target might 
prefer different portfolios if their risk aversion differs from one another. Cited in the commented paper, Ballestero (2001) has proposed a MCDA portfolio selection model in which $\mathrm{M}-\mathrm{V}$ and risk aversion are articulated.

\section{The case of fund performance analysis}

In Sect. 4.2 of the paper, corporate performance analysis but not fund performance analysis is examined. As to funds, we wonder if classical performance measures unrelated to preferences (e.g., Sharpe's ratio, Graham and Harvey leverage) are more suitable than preference-based outranking methods. It is rather obvious that manager's preferences should not be used instead of investor's preferences, which differ from an investor to another. On this subject, Arrow's impossibility theorem or Arrow's paradox states that constructing social preferences from individual preferences is logically impossible. More precisely, no rank order voting system can convert the ranked preferences of individuals (investors in our case) into a community-wide complete and transitive ranking when the number of alternatives is 3 or more. See Geanakoplos (2001).

\section{Remark}

As readers are especially interested in mathematical developments, we suggest to add a mathematical section in which particular aspects of the paper might be algebraically or numerically addressed.

\section{References}

Arrow KJ (1965) Aspects of the theory of risk-bearing

Ballestero E (2001) Stochastic goal programming: a mean-variance approach. Eur J Oper Res 131(3):476481

Copeland TE, Weston JF (1988) Financial theory and corporate policy. Addison-Wesley, Reading

Doumpos M, Zopounidis C (2010) A multicriteria decision support system for bank rating. Decis Support Syst 50(1):55-63

Doumpos M, Zopounidis C (2011) A multicriteria outranking modeling approach for credit rating. Decis Sci 42(3):721-742

Geanakoplos J (2001) Three brief proofs of arrow's impossibility theorem. Yale Cowles Foundation discussion paper (1123RRR)

Konno H, Yamazaki H (1991) Mean-absolute deviation portfolio optimization model and its applications to Tokyo Stock Market. Manag Sci 37(5):519-531

Saaty TL, Ozdemir MS (2003) Why the magic number seven plus or minus two. Math Comput Model 38(3):233-244

Sun S, Lu WM et al (2005) A cross-efficiency profiling for increasing discrimination in data envelopment analysis. Inf Syst Oper Res 43(1):51 